\title{
Efficacy of EGFR TKI Targeted Therapy versus TKI in Combination with Chemotherapy in Non-Small-Cell Lung Cancer
}

\author{
Alaina Johnston ${ }^{1}$, Takefumi Komiya ${ }^{2}$ \\ ${ }^{1}$ Indiana University School of Medicine; ${ }^{2}$ Parkview Health, Department of Oncology
}

\section{Background and Hypothesis:}

Non-Small Cell Lung Cancer (NSCLC) constitutes the largest proportion of lung cancers and is the foremost cause of mortality associated with cancer around the world. Of patients with nonsmall cell lung cancer, approximately $15 \%$ of Caucasians and $30 \%$ of Asians have activating mutations in the epidermal growth factor receptor (EGFR) gene. Numerous studies have indicated increased progression free survival after treatment with tyrosine kinase inhibitors (TKI). However, the most efficacious combination of drugs, whether TKIs, chemotherapy, or anti-angiogenesis, is still unknown. This literature review will be constructed to determine whether tyrosine kinase inhibitor targeted therapy in combination with chemotherapy or antiangiogenesis drugs is more effective in prolonging progression free survival in EGFR-mutated NSCLC as opposed to tyrosine kinase inhibitor targeted therapy alone.

\section{Project Methods:}

The methodology of this proposed literature review is an online search of PubMed, Journal of Clinical Oncology, and important scientific conferences. The treatment interventions consist of tyrosine kinase inhibitor targeted therapy in combination with chemotherapy and tyrosine kinase inhibitor targeted therapy alone. The projected outcome is an increase in progression free survival and overall survival. The proposed data analysis consists of constructing a forest plot in order to display the results.

\section{Results:}

The results are expected to support the hypothesis, that combination treatment with TKI and chemotherapy will allow patients with an opportunity to prolong their progression-free survival. The forest plot is expected to indicate a progression-free survival hazard ratio that favors combination therapy. P-values will be included to indicate statistically significant results.

\section{Conclusion and Potential Impact:}

The importance of finding the answer to this hypothesis is that it will improve treatment outcomes for patients with NSCLC. It will provide patients with a strong alternative to chemotherapy or tyrosine kinase inhibitor therapy alone. Most importantly, it will provide patients with an avenue to increase their progression-free survival. 